
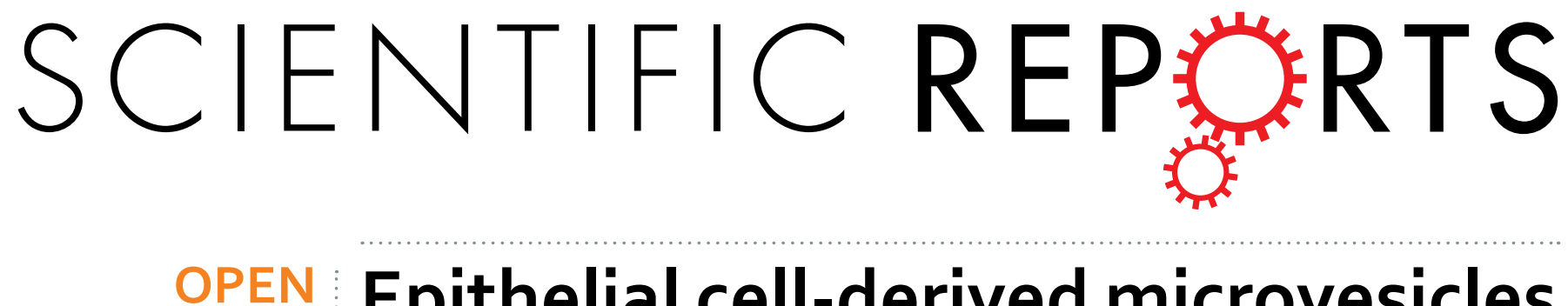

\title{
Epithelial cell-derived microvesicles activate macrophages and promote inflammation via microvesicle- \\ containing microRNAs
}

Received: 11 July 2016

Accepted: 27 September 2016

Published: 12 October 2016

\author{
Heedoo Lee ${ }^{1, *}$, Duo Zhang ${ }^{1, *}$, Ziwen Zhu ${ }^{1}$, Charles S. Dela Cruz ${ }^{2} \&$ Yang Jin $^{1}$
}

Intercellular communications between lung epithelial cells and alveolar macrophages play an essential role in host defense against acute lung injury. Hyperoxia-induced oxidative stress is an established model to mimic human lung injury. We show that after hyperoxia-associated oxidative stress, a large amount of extracellular vesicles (EVs) are detectable in bronchoalveolar lavage fluid (BALF) and culture medium of lung epithelial cells. Microvesicles (MVs), but not exosomes (Exos) or apoptotic bodies (Abs), are the main type of EVs found in the early stages after hyperoxia. Among all the MV compositions, small RNAs are altered the most significantly after hyperoxia-associated oxidative stress. We further confirmed that hyperoxia up-regulates the levels of certain specific miRNAs in the epithelial cell-derived MVs, such as the miR-320a and miR-221. Functionally, the hyperoxia-induced epithelial MVs promote macrophage activation in vitro and facilitate the recruitment of immunomodulatory cells in vivo detected in BALF. Using MV as a cargo, delivery of the specific miRNA-enriched epithelial MVs (miR-221 and/or miR-320a) also triggers macrophage-mediated pro-inflammatory effects. Collectively, epithelial cell-derived MVs promote macrophage-regulated lung inflammatory responses via MV-shuttling miRNAs.

In the past decade, emerging evidence suggests that extracellular vesicles (EVs) play an essential role in intercellular cross-talks ${ }^{1,2}$. Accumulating reports have demonstrated that EV-shuttling molecules, including proteins, RNAs, microRNAs (miRNAs) and lipids, potentially exerting crucial physiological and pathological functions in tumorigenesis ${ }^{3}$ and metastasis ${ }^{4}$. These novel discoveries shed light on the development of novel tumor markers and new therapies ${ }^{5,6}$. However, less studies focus on the pathogenesis of non-cancer-related diseases. For example, very little is known on the role of EVs in lung diseases.

Acute respiratory distress syndrome (ARDS) is a devastating disease featured with profound lung injury and characterized by the disruption of the epithelial and endothelial barrier, flooding of the alveolar compartment with protein-rich fluids, and recruitment of neutrophils into the alveolar space ${ }^{7,8}$. Numerous types of cells reside in the lungs and how they communicate with each other is largely understood. Human lung epithelium has a large surface area and epithelial cells play a key role in innate immunity against noxious insults and are involved in the recruitment of various inflammatory mediators into the alveolae $e^{9,10}$. These epithelial cells are important in the pathogenesis and resolution of acute lung injury (ALI $)^{11,12}$. Epithelial cells respond to sterile injury and infection and activate alveolar macrophages ${ }^{13-15}$. Activated alveolar macrophages, along with other lung cells (which ones?), regulate leukocyte influx through the production of proinflammatory cytokines, chemokines and other factors that modulate chemokine gradients and effect leukocyte migration ${ }^{16-18}$. However, how epithelial cells communicate with alveolar macrophages remains unclear. Despite that EVs have been thought as potential candidates in mediating cell-cell crosstalks in cancer metastasis and other disease processes ${ }^{19,20}$, not much is known about the role of EVs in the lung. Currently, the EV generations in the lungs, the release of EVs from lung cells, and the functions of lung-derived EVs are poorly delineated, impeding the development of novel targets for novel diagnosis and therapy of lung injury.

${ }^{1}$ Division of Pulmonary and Critical Care Medicine, Department of Medicine, Boston University, Boston, MA 02118, USA. ${ }^{2}$ Section of Pulmonary, Critical Care and Sleep Medicine, Yale University School of Medicine, New Haven, CT 06519, USA. *These authors contributed equally to this work. Correspondence and requests for materials should be addressed toY.J. (email: yjin1@bu.edu) 
The contents, size and membrane composition of EVs are heterogeneous based on the cellular sources and environmental stimuli ${ }^{14,21,22}$. According to the International Society of Extracellular Vesicles, three main subgroups of EVs have been defined based on the size, the membrane compositions and the mechanisms of formation $^{23}$. Apoptotic bodies (ABs) (500-2000 $\mathrm{mm}$ diameter) are formed by plasma membrane blebbing in the process of undergoing apoptosis. Microvesicles (MVs) $(50-500 \mathrm{~nm})$ are the second subgroup, comprising of different sized vesicles directly protruding from plasma membranes. Exosomes (Exos) are the smallest subgroup (approximately 30-100 $\mathrm{nm}$ in diameter) and are released from cells after multivesicular bodies (MVBs) fuse with the plasma membrane ${ }^{20}$. A subset of proteins derived from parent cells are often detectable in EVs. Surfactant proteins (SPs) can be detected in the EVs derived from lung epithelial cells ${ }^{24}$. Interestingly, MVs and exosomes also carry distinct proteins that can be found only in one of the two. For instance, vesicle-associated membrane protein 3 (VAMP3) can be found in the MVs generated from melanoma cells, while transferrin receptors are highly enriched in exosomes, but missing in the $\mathrm{MVs}^{25}$. However, no single marker can uniquely identify each subgroup of EVs. Proteins which often have been used as markers of EVs but not specific to either exosomes or MVs, include: tetraspanins such as CD9, CD63, CD81 and CD82 $2^{26}$.

EV-containing RNAs were first described in EVs derived from murine stem cells ${ }^{27}$. Despite the smaller sizes of EV intact messenger RNAs (mRNAs), long non-coding RNAs (lncRNAs), ribosomal RNA (rRNA) and the fragments of these intact RNA molecules have all been identified in EVs ${ }^{23,28,29}$. MiRNAs are 20-22 nt small non-coding RNA molecules which exert essential regulatory functions on their target genes ${ }^{30}$. Many types of miRNAs have been identified in $\mathrm{EVs}^{31-33}$. However, detailed characterizations on the miRNA profiles in exosomes or MVs require further investigation, particular in non-tumor cells. Recent studies have indicated that the number of copies of "highly upregulated" miRNAs found in tumor cells is in fact very low in each individual exosome detected in plasma ${ }^{34}$. This raises the question of whether these exosomal miRNAs indeed can have physiological or pathological effects on their target cells ${ }^{35}$. That said, no studies have explored the miRNAs contained in MVs. Therefore, before performing any further investigations that is focused on the role of EV-miRNAs, we need to first determine the type of EVs that is generated in lungs after noxious insults and determine whether these stimuli-induced EVs contain miRNAs. In this study, we will characterize the generation of EVs, their category/ type based on size, composition and function in lung epithelial cells, as well as whether EVs play a role in the crosstalk between epithelial cells and alveolar macrophages.

\section{Results}

Characterization of the EV generation and release in lung BALF and epithelial cells. We first determined the generation and release of EVs in mouse lungs. We obtained broncho-alveolar lavage fluid (BALF) and isolated EVs using ultracentrifugation as previously described ${ }^{23}$. Based on the sizes of EVs that were determined using DLS (spell out as well), we identified three types of EVs and the range of sizes as shown in Fig. 1A. The percentages of the three types of EVs are illustrated in Fig. 1B. Of all the EVs, $57 \%$ were MVs, $20 \%$ were ABs and $23 \%$ were Exos (Fig. 1B). Hyperoxia significantly augmented the levels of MVs in BALF, comparing to ABs or Exos (Fig. 1C). We previously have shown that majority of EVs are derived from lung epithelial cells after hyperoxia ${ }^{14}$. Therefore, to obtain a convenient cellular model to study the underlying mechanisms, we exposed Beas2B human lung epithelial cells to hyperoxia. Similarly, majority of Beas2B epithelial cell-derived EVs fell into the ranges of MVs (53\%) (Fig. 1D). The ABs, MVs and Exos generated from Beas2B cells were visualized under electric microscopy (Fig. 1E). To confirm this observation, we further isolated mouse lung primary epithelial cells and exposed them to hyperoxia. Similar results were obtained. As shown in Fig. 1F, again, 63\% of EVs were MVs, while $18 \%$ and $19 \%$ of EVs were ABs and Exos, respectively (Fig. 1F). Previously, we have shown that the BALF EVs are mainly derived from the epithelial cells in the presence of hyperoxia ${ }^{14}$.

Characterization of the EV compositions in lung BALF and epithelial cells. MiRNA compositions in hyperoxia-induced MVs were analyzed using microRNA arrays. Heat map is shown on Fig. 2A. Hyperoxia augmented the level of MV-protein, MV-RNA (Fig. 2B, left and middle panels). After normalization with the elevated protein, MV-RNA after hyperoxia remains significantly higher than the MV-RNA in RA (Fig. 2B, right panel). Interestingly, hyperoxia failed to alter the level of Exo-protein, Exo-RNA (Fig. 2C, left and middle panels). After normalization with Exo-protein, Exo-RNA after hyperoxia remains at the same level comparing to those obtained in RA (Fig. 2C, right panel). We next evaluate the MV-RNA and Exo-RNA using human lung epithelial cells. As shown in Fig. 2D,E, hyperoxia augmented the level of MV-protein and MV-RNA which were derived from Beas2B cells (Fig. 2D, left and middle panels). After normalization with the elevated protein, Beas2B cell-derived MV-RNA after hyperoxia remains significantly higher than MV-RNA in RA (Fig. 2D, right panel). Despite that hyperoxia augmented the level of Exo-protein and Exo-RNA which were derived from Beas2B cells (Fig. 2E, left and middle panels), after normalization with the elevated protein, Beas2B cell-derived Exo-RNA after hyperoxia remains at the same level compared to those obtained in RA (Fig. 2E, right panel). This observation is consistent with those results obtained using BALF.

Quantification of the MV counts in the absence or presence of hyperoxia and determination of MV-RNA or MV-protein levels in each MV. To better quantify the amount of MVs induced by oxidative stress, we first established a method to quantify the MV counts. Initially, MVs were fluorescently labeled with protein-carboxy-fluorescein-succinimidyl ester (CFSE) as previously described ${ }^{36}$, verifying that vesicle numbers were increased in a dose-dependent manner (Fig. 3A). A standard curve between the pre-set particle numbers and the measured particle counts using DLS was set as detailed in the Material and Methods (Fig. 3B). Therefore, the MV numbers in a given sample can be indirectly calculated based on the DLS counts using the standard curve. Using this method, we assessed the MV numbers in the absence and presence of hyperoxia (Fig. 3C). Total MV protein amount is shown in Fig. 3D. Next, we determined the amount of MV protein per each MV (Fig. 3E). 
A

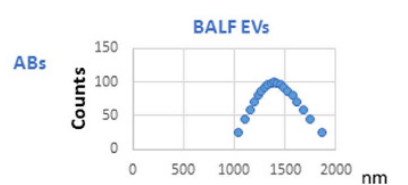

B

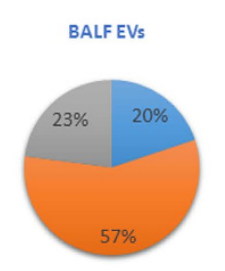

C

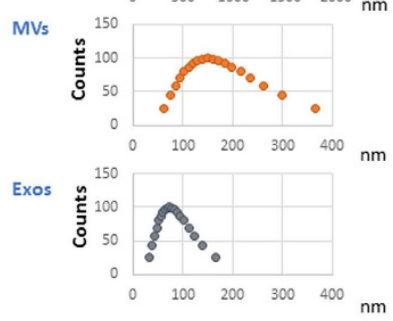

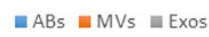

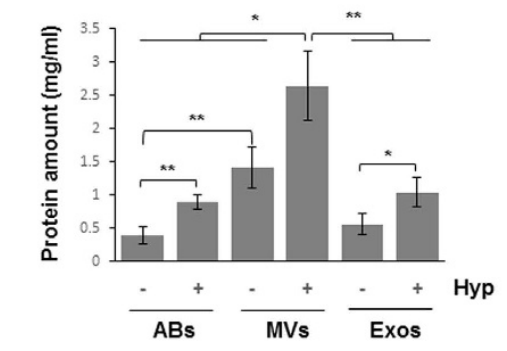

D

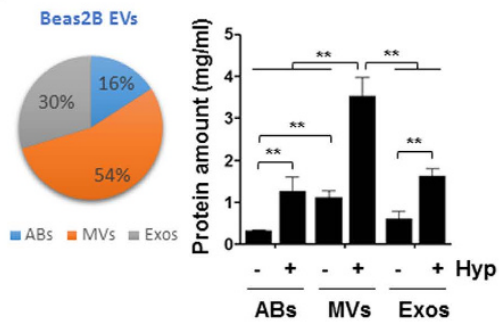

E
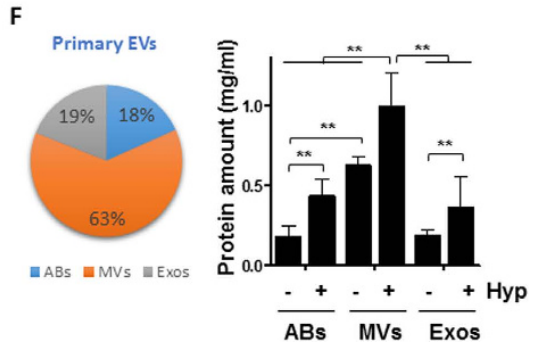

Figure 1. Characterization of the extracellular vesicles (EVs) generated from BALF and lung epithelial cells. $(\mathbf{A}, \mathbf{B})$ Three types of EVs were isolated from mouse broncho-alveolar lavage fluid (BALF), including apoptotic bodies (ABs), microvesicles (MVs), and exosomes (Exos). The sizes of the isolated EVs were measured using Dynamic Light Scattering (DLS) (A). Pie graph indicates the percentages of each type of EVs derived from BALF (B). (C) Mice were exposed to hyperoxia for 3 days, followed by isolation of three types of EVs from BALF. Amount of EVs were shown in the bar graph. $\mathrm{n}=4$ mice per group. Bease2B cells $(\mathrm{D})$ and primary epithelial cells (F) were exposed to hyperoxia for 2 days, followed by isolation of EVs. Left panels show pie graphs indicating the percentages of each type of EVs. Right panels show the protein amounts of EVs in bar graphs. (E) Three types of EVs were isolated from Beas2B cells, and visualized using transmission electron microscope (TEM). Data represent mean \pm SD of three independent experiments with identical results.

A

BALF MVS

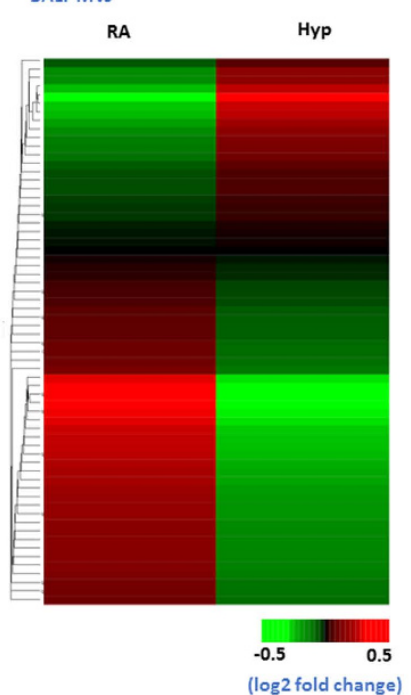

BALF MVS

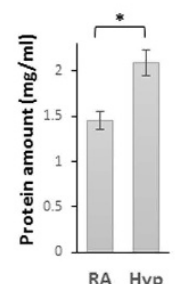

D
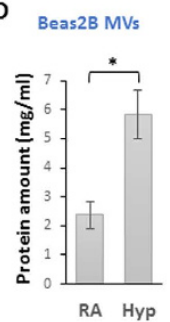
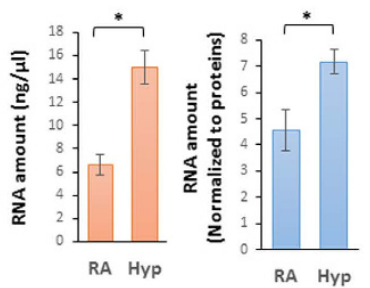

RA Hyp

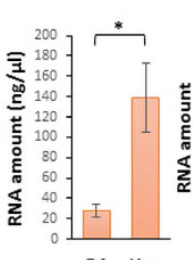

RA Hyp

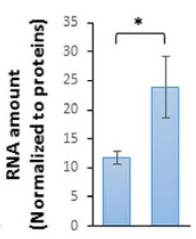

RA Hyp BALF Exos
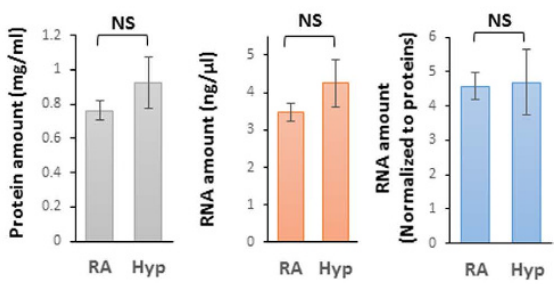

E Beas2B Exos
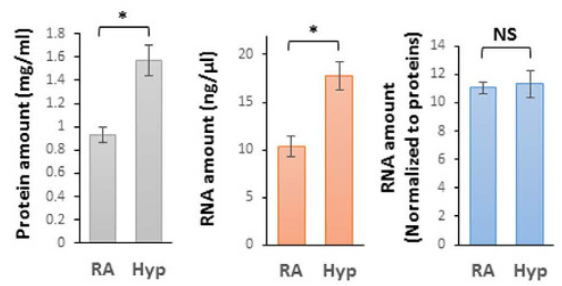

Figure 2. RNAs are accumulated in MVs in response to hyperoxia. (A) Heat map of the miRNA profiles in the MVs which were isolated from BALF. (B-E) Mice and Beas2B cells were exposed to hyperoxia for 2 days. MVs and Exos were isolated from BALF $(\mathbf{B}, \mathbf{C})$ and cultured medium of the Bease2B cells $(\mathbf{D}, \mathbf{E})$ followed by isolation of total RNAs from the MVs or Exos. Proteins, RNAs, and normalized RNA using protein amounts were shown. Data represent mean $\pm S D$ of three independent experiments with the similar results. 
A

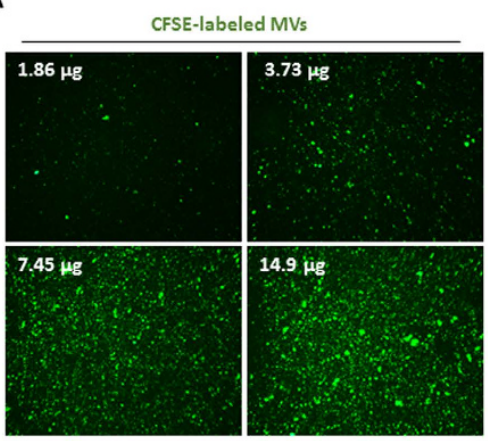

B

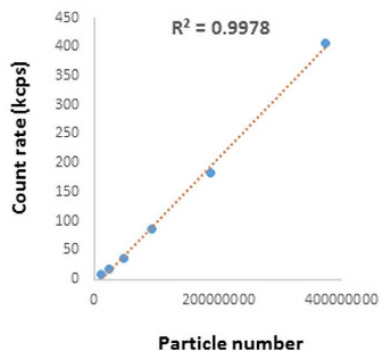

c

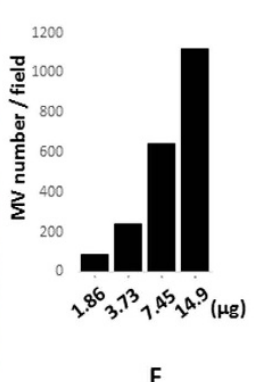

F

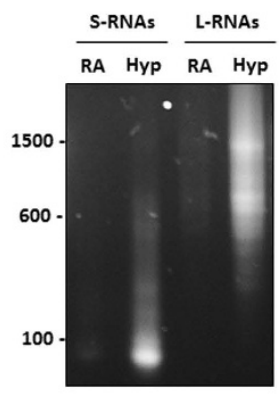

D

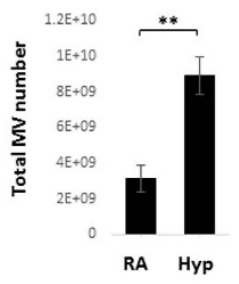

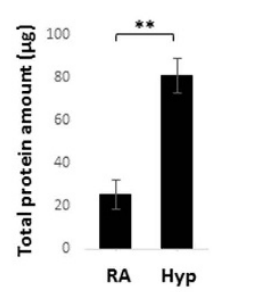

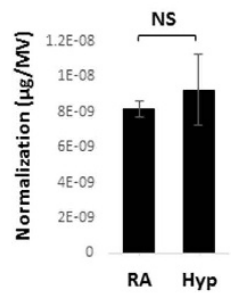

G

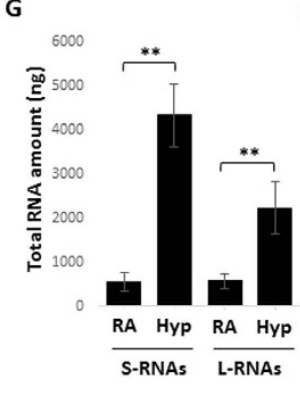

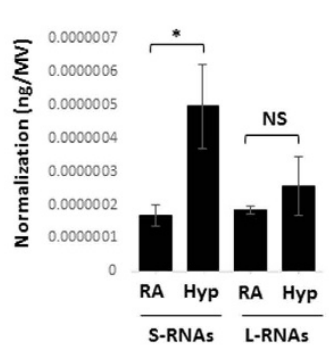

Figure 3. Quantification of RNA and protein amount in each MV. (A) MVs were isolated from Beas2B cells, and labeled with carboxyfluorescein succinimidyl ester (CFSE). Indicated protein amount of MVs were fixed and visualized using fluorescence microscope. Vesicles in field (20x magnification) were counted using NIH ImageJ software (right panel). (B) A liner calibration standard curve (particle number vs. count). The counts of particles with the pre-set numbers were measured using DLS, followed by generation of a linear calibration curve $\left(R^{2}=0.9978\right)$. For $A$ and $B$, data representative of two independent experiments. $(\mathbf{C}-\mathbf{E})$ MVs were isolated from the Beas2B cells after hyperoxia. Total MV number $(\mathbf{C})$ total MV protein amount (D) and protein amount per MV (total MV protein/MV counting numbers) (E) were shown. (F-H) Large and small RNAs were purified from the isolated MVs and subjected to electrophoresis using agarose gel, as illustrated in (F). Total RNA amount is shown in (G). RNA amount per MV (total MV RNA/MV counting numbers) are shown in (H). For $\mathbf{C}-\mathbf{H}$, data represent mean $\pm \mathrm{SD}$ of three independent experiments.

Interestingly, the same amount of protein was found in the MVs generated in the absence or presence of hyperoxia (Fig. 3E). After hyperoxia, the MV RNAs were subjected to agarose gel fraction (Fig. 3F). Based on their number of base pairs, small size and large size of MV-RNAs after RA or hyperoxia are shown in Fig. 3G. After normalized with the MV counts, we found that in a given MV, RNAs with smaller sizes were increased much more robustly compared with RNAs with larger number of base pairs (Fig. $3 \mathrm{H}$ ). This observation prompted us to focus on MV-miRNAs which only have approximately 20-22 base pairs of nucleotides.

Hyperoxia-induced, epithelial cell-derived MV promotes immunomodulatory cell recruitment in vivo and macrophage activation in vitro. Macrophage cell counts were increased in BALF obtained from the mice that were exposed to hyperoxia in a time-dependent manner (Fig. 4A), compared to those that were exposed to RA. We isolated the hyperoxia-induced MVs from BALF and treated the C57BL/6 mice via inhalation. As shown in Fig. 4B, inhaled MVs significantly enhanced the macrophage cell counts in BALF, particularly in the presence of hyperoxia (Fig. 4B). We also isolated the hyperoxia-induced, human epithelial cell (Beas2B) -derived MVs and treated the human THP1 macrophage in vitro using these isolated MV. Macrophage migration was markedly stimulated after being treated with the Beas2B-derived MVs in vitro (Fig. 4C). Furthermore, TNF- $\alpha$, IL- $1 \beta$ and IL-10 secretions from THP1 macrophages were measured. Significantly enhanced TNF- $\alpha$ and IL-1 $\beta$ secretions after exposure to hyperoxic MVs were observed (Fig. 4D, left and middle panels).

Hyperoxia augments specific miRNA levels in MVs and the hyperoxia-upregulated miRNAs activate macrophages in a synergistic manner. We next determined the miRNA compositions in hyperoxic MVs obtained from BALF after hyperoxia. A number of upregulated miRNAs were identified in hyperoxic MVs using miRNA profiling, as described in Materials and Methods. Figure 5A shows 8 miRNAs whose levels were significantly elevated in BALF MVs after hyperoxia (Fig. 5A). We chose three specific miRNAs (miR320a, miR-221, and miR-342) and further confirmed their enhanced expression in Beas2B epithelial MVs after hyperoxia (Fig. 5B). Similar observations were found using mouse lung primary epithelial cells (Fig. 5C). To illustrate the functions of these hyperoxia-induced miRNAs, we transfected THP1 macrophages with miR-221 and/or miR-320a mimics. Macrophage migration assays were performed as described in Materials and Methods. Both miR-221 and miR-320a promoted macrophage migration significantly. More interestingly, miR-221 and miR-320a robustly exerted synergistic effects on macrophage migration (Fig. 5D). We further found that miR-221 
A

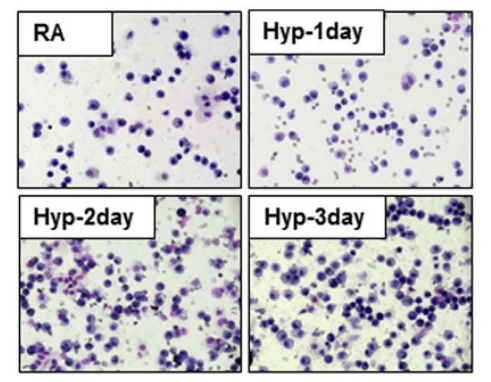

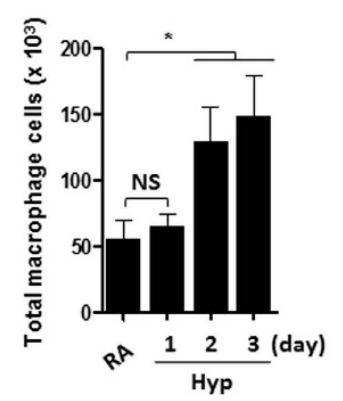

C

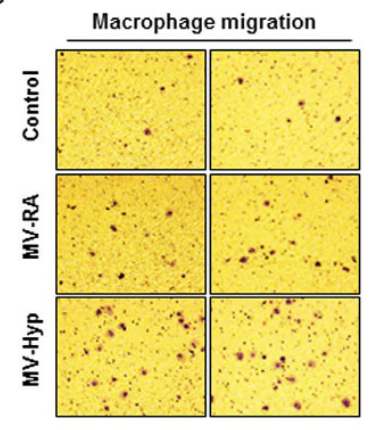

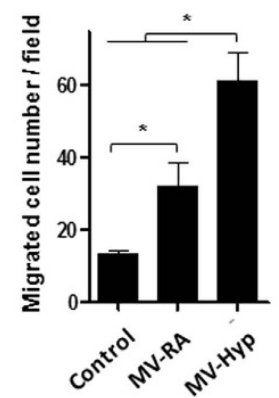

B
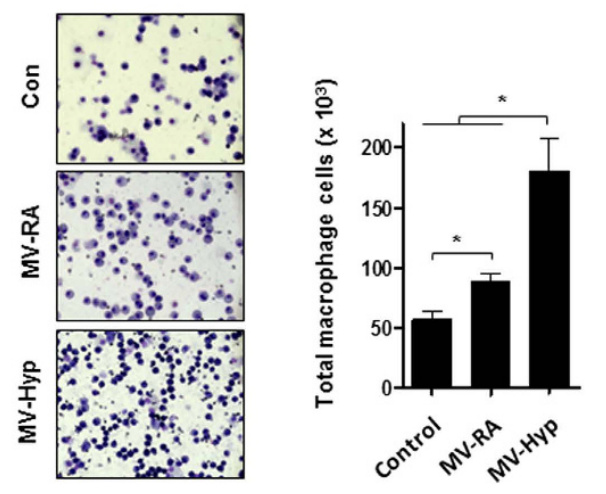

D
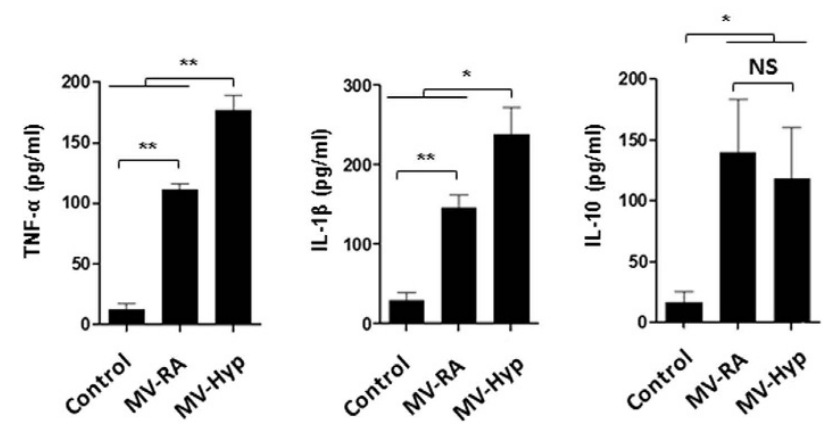

Figure 4. Lung epithelial cell-derived MVs induce macrophage migration and cytokine release. (A) Mice were exposed to hyperoxia for the designated time period. BALF cells were stained with hematoxylin and eosin (H\&E), followed by cell counting. $n=4$ mice per group. (B) BALF-derived MVs were isolated from mice 3 days after hyperoxia. BALF cells were stained with $\mathrm{H} \& \mathrm{E}$ and analyzed $24 \mathrm{~h}$ after exposed to the RA-MVs and hyperoxia-induced MVs $(10 \mu \mathrm{g} / 50 \mu \mathrm{l}$ per mice) intranasally. $\mathrm{n}=4$ mice per group. (C) MVs were isolated from the Beas2B cells 2 days after hyperoxia. THP1 macrophages were treated with the Beas $2 \mathrm{~B}$ cell-derived MVs $(5 \mu \mathrm{g} / 500 \mu \mathrm{l}$ per well). After $16 \mathrm{~h}$, transwell migration assay of THP1 macrophages was performed, as described in Material and Methods. Representative images of migrated cells (left panel) and quantification graphs (right panel) are shown. (D) THP1 macrophage were treated with isolated MVs ( $5 \mu \mathrm{g} / 500 \mu \mathrm{l}$ per well) described in (B). After $16 \mathrm{~h}$, TNF- $\alpha$, IL-1 $\beta$, and IL-10 were analyzed using ELISA. For C and D, data represent mean \pm SEM of three independent experiments.

and miR-320a up-regulated MMP9 (Fig. 5E), a molecule which is known to be involved in macrophage migration $^{37-39}$. Similarly, a synergistic effect between miR-221 and miR-320a on macrophage-derived TNF- $\alpha$ secretion was also observed (Fig. 5F), in addition to miR-221 or miR-320a inducing TNF- $\alpha$ secretion individually (Fig. 5F). Consistently, the well-known mechanistic pathway NF- $\kappa \mathrm{B}$ pathway components were targeted and up-regulated by miR-221 and miR320a (Fig. 5G).

MVs activate macrophages via MV-shuttling miRNAs. In Fig. 5, we demonstrated that hyperoxia augmented certain specific miRNAs in the epithelial cell-derived MVs. Furthermore, these miRNAs synergistically activated macrophages. We next ask whether these "pro-inflammatory" miRNAs can be delivered to the recipient cells in a MV-shuttling manner. We isolated and cultured the mouse primary lung epithelial cells. MVs were isolated from these cells and selected miRNAs were directly transfected into isolated MVs as described in Materials and Methods. Here we chose miR-221 and miR-320a as examples to be consistent with the above data. MVs with enhanced miR-221 and/or miR-320a were used to treat mouse BMDMs, as illustrated in Fig. 6A. We first confirmed that MV-shuttling RNAs were successfully delivered into the recipient cells using labeled MV-RNAs (Fig. 6B), as described in the Materials and Methods. BMDMs treated with MVs with the enhanced miR-221 and/ or miR-320a augmented BMDM migrations. More importantly, MVs which contain both the enhanced miR-221 and miR-320a carried the strongest effects on promoting macrophage migration (Fig. 6C). TNF- $\alpha$ secretion and MMP-9 level/activity were also enhanced in the same pattern, i.e. MV-miR-221 and MV-miR-320a synergistically up-regulated MMP level and activities, as well as TNF- $\alpha$ secretion from BMDMs (Fig. 6D,E, respectively). Consistent results were found on the expression of NF- $\kappa B$ pathway components (Fig. 6F).

\section{Discussions}

Emerging evidence suggests that EVs potentially can serve as novel targets for the development of therapeutic and diagnostic reagents. While intensive studies have focused on the role of EVs in cancer metastasis, the generation, regulation and function of EVs in lung diseases remain largely unexplored. EVs describe a group of heterogeneous vesicles which have different, characteristics, mechanisms of formation and functions $\mathbf{s}^{5,19,22}$. To develop 
A

\begin{tabular}{|c|c|c|}
\hline \multirow{2}{*}{ miRNA } & \multicolumn{2}{|c|}{ MVs } \\
\cline { 2 - 3 } & Fold increase & Basal level \\
\hline hsa-mir-92a-3p & 1.23 & 39.15 \\
\hline hsa-mir-320a & 1.30 & 56.57 \\
\hline hsa-mir-33a-5p & 1.33 & 1.25 \\
\hline hsa-mir-221-3p & 1.41 & 42.62 \\
\hline hsa-mir-145-5p & 1.44 & 1.82 \\
\hline hsa-mir-342-3p & 1.47 & 9.37 \\
\hline hsa-mir-10a-5p & 1.52 & 1.3 \\
\hline hsa-mir-422a & 1.84 & 1.6 \\
\hline
\end{tabular}

B

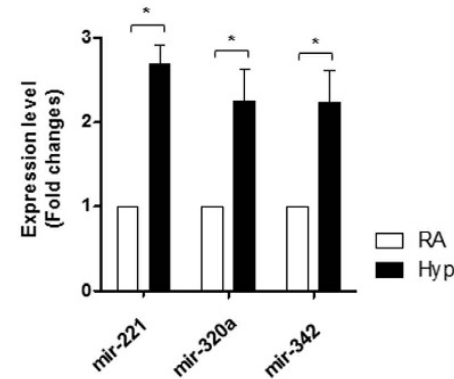

$\mathbf{F}$

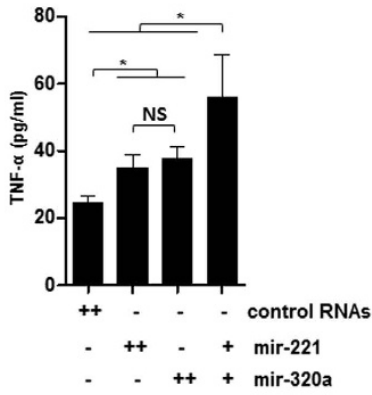

C

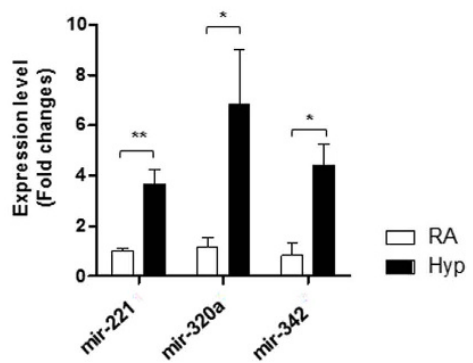

G

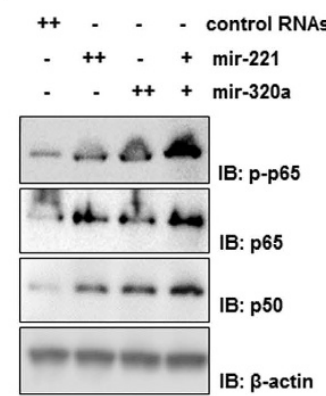

Figure 5. BALF- and lung epithelial cell-derived MVs contain pro-inflammatory miRNAs. (A) Elevated miRNAs derived from BALF MVs after hyperoxia. The table was generated from miRNA microarray data as shown in Fig. 2A. (B,C) MVs were isolated from Beas2B cells (B) and primary epithelial cells (C). RNA was isolated from the MVs and quantified using the real-time quantitative RCR (qPCR). Individual miRNA expression levels were shown in bar graphs (D). Transwell migration assay of THP1 macrophages was performed after treated with mir-221 and/or mir-320a mimics which were transfected using lipofectamine 2000, as described in Material and Methods. (E-G) THP1 macrophages were treated with mir-221 and/or mir320a mimics. After 16h, MMP-9 levels (E) and TNF- $\alpha$ levels (F) from the culture medium were analyzed using gelatin zymography and ELISA, respectively. Western blot analysis was performed using total cell lysates with the indicated antibodies $(\mathbf{G})$. For $(\mathbf{B}-\mathbf{F})$ data represent mean \pm SD of three $(\mathbf{D}-\mathbf{F})$ or four $(\mathbf{B}, \mathbf{C})$ independent experiments. For $(\mathbf{G})$ data represent two independent experiments with the similar results. Unprocessed original scans of gel and blots are shown in Suppl. Fig. 1.

novel therapeutic agents and diagnostic markers, the types of EVs and the compositions of EVs, along with their dynamic changes in the pathogenesis of lung diseases, require a complete characterization. Our reports, for the first time, demonstrated that after oxidative stress, MVs, rather than exosomes, are the main types of EVs that are generated by live lung epithelial cells.

Small RNAs are the main MV-components that change the most significantly when compared with proteins or large RNAs during hyperoxia-associated oxidative stress (Fig. $3 \mathrm{H}$ ).

Our data further identified the MV-miRNA profiles which have been significantly altered after hyperoxia (Fig. 2A). Using realtime PCR, we confirmed that a number of miRNAs robustly was increased in the epithelial cell-derived MVs after exposure to hyperoxia (Fig. 5B,C). Interestingly, despite the rapidly growing interests that have focused on the potentials of EV-miRNAs to serve as biomarkers or therapeutic targets, the number of copies of "highly upregulated" miRNAs found in tumor cells remains at a very low level in each individual exosome detected in the plasma ${ }^{34}$. Therefore, whether the miRNAs are the main functional components in exosomes is ambiguous $^{23,40}$. Our studies show that after hyperoxia, the main form of epithelial cell-derived EVs is MVs rather than exosomes. First of all, a relatively high copy numbers of MV-miRNAs were induced by hyperoxia (Fig. 5B,C). More importantly, a group of miRNAs, but not only one single miRNA, were upregulated in MVs (Figs 2A and 5A). Our functional studies confirmed that many of these upregulated MV-miRNAs carried functional effects and promoted TNF- $\alpha$ and IL- $1 \beta$ secretions from macrophages (Fig. 6C-F). Robust synergistic effects were observed among the upregulated MV-miRNAs, suggesting crucial roles of epithelial cell-derived MV-miRNAs in mediating macrophage activation. Our studies illustrate the complexity of developing diagnostic/therapeutic targets using EV or EV compositions. First, the pattern of different type of EVs involved in the pathogenesis requires a detailed characterization. This process is probably stimulation-dependent and cell type-dependent. Second, multiple compositions in certain type of EVs involved in the disease process needs to be delineated. Third but not the last, the synergistic effects of different EV compositions are to be addressed.

The underlying mechanisms to generate MVs and exosomes are clearly different. MVs are formed via direct protruding of cell plasma membrane while exosomes are much smaller vesicles which are generated after multivesicular bodies (MVBs) fuse with the plasma membrane ${ }^{20}$. GW182 and AGO2, two main components of the RNA-induced silencing complex (RISC) that congregate with endosomes and $\mathrm{MVBs}^{41}$. Exosomes have been 
A

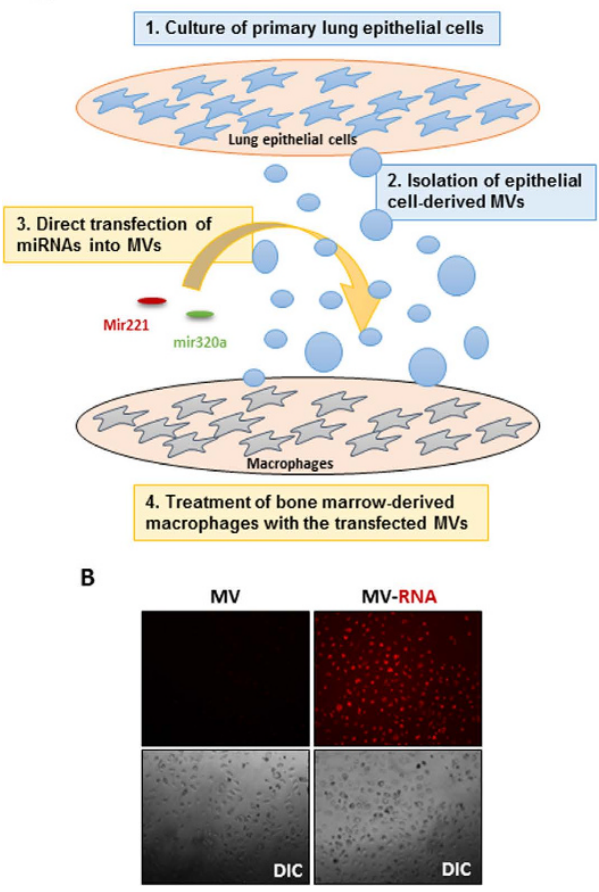

C

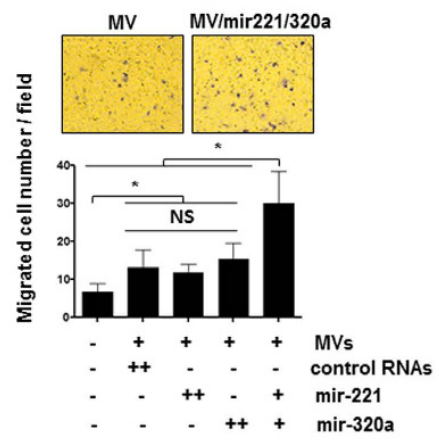

E

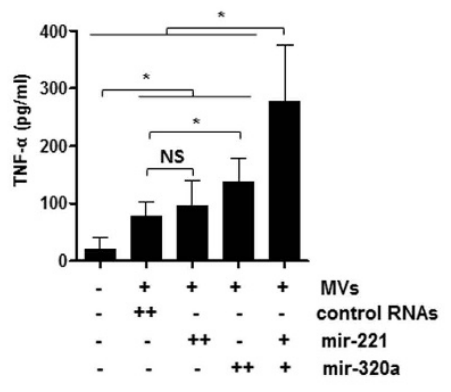

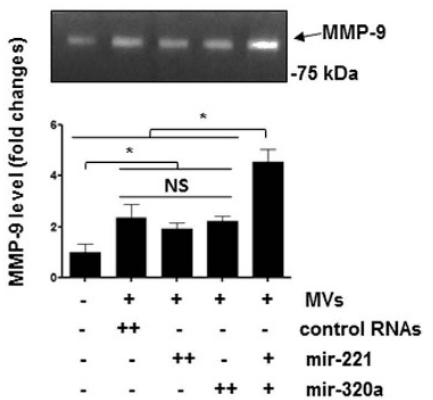

$\mathrm{F}$

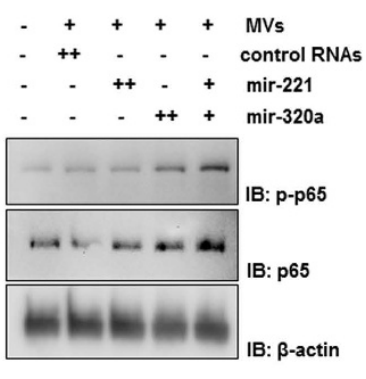

Figure 6. Synergistic effects of MV-containing mir-221 and 320a on the macrophage migration and cytokine secretion. (A) Schematic illustration of the functional analysis of MV-containing miRNAs on the macrophage activation. MVs were isolated from the mouse primary lung epithelial cells and directly transfected with mir-221 and/or 320a as described in Material and Methods. Mouse BMDMs were treated with the transfected MVs and the macrophage migration and cytokine secretion were measured. (B) MV-containing RNAs derived from the primary epithelial cells were labeled with acridine orange and incubated with the mouse BMDMs. After 16h, MV-RNAs were visualized using fluorescent microscope. The MV internalization was shown in the upper panels. Differential Interference Contrast (DIC) cell images were shown in the lower panels. (C-F) Mouse BMDMs were treated with the transfected MVs $(5 \mu \mathrm{g} / 500 \mu \mathrm{l}$ per well) described in (A). After 16 h, BMDM migration was measured using the transwell assay, as described in Material and Method (C). Representative images of migrated cells (upper panel) and quantification graphs (lower panel) are shown. MMP-9 levels (D) and TNF- $\alpha$ levels (E) from the culture medium were analyzed using gelatin zymography and ELISA, respectively. Western blot analysis was performed using total cell lysates $(\mathbf{F})$ with the indicated antibodies. For $(\mathbf{C}-\mathbf{E})$ data represent mean $\pm S D$ of three independent experiments. For $(\mathbf{B}, \mathbf{F})$ data represent two independent experiments with the similar results. Unprocessed original scans of gel and blots are shown in Suppl. Fig. 1.

shown to contain GW182 but not AGO2 ${ }^{41}$. Therefore, GW182-associated miRNAs are more likely being found in exosomes, possibly in association with only certain types of disease process.

In addition to miRNAs, the content of MVs and exosomes is highly enriched with a variety of other components, including the proteins derived from the parent cells $s^{21,42}$. However, it is unclear whether all of these components indeed carry significant biological functions or simply represent debris from cell damage. Previously, we have shown that certain protein components in the epithelial cell-derived EVs can function as a signal transmitter, such as through caspase- $3^{14}$. In the previous report, we did not clarify which type of EVs consists of caspase-3. Based on the sizes, presumably, the caspase- 3 also resides in epithelial cell-derived MVs. Exosomal protein is likely at relatively smaller size and consists of more fragments of proteins.

Our data suggest that the number of small RNAs including miRNAs increased more dramatically after hyperoxia, compared with large RNAs and proteins; therefore, this report did not specifically explore the potential functions of MV-proteins. Furthermore, this current study did not consider the effects of MVs on macrophage activation via cell surface receptors. Instead of engulfing all the MV compositions, macrophage activation potentially can be triggered via the formation of macrophage surface receptor-MV complex, followed by a signaling cascade to activate macrophages. Detailed characterizations of the proteins presented on the surface of epithelial cell-derived MVs after oxidative stress require further exploration. Another remaining limitation involving our studies is the difficutly in determining the minimum amount of MVs or MV-miRNAs that are required to activate the recipient macrophages, particularly in vivo. Presumably, this question is largely affected by how many different miRNAs and proteins are altered by each stimulation. Therefore, this minimum "dose" of MV-miRNAs may also be cell type and stimulation dependent.

In summary, our report finds that after hyperoxia-associated oxidative stress, live lung epithelial cells release a significant amount of MVs, rather than Exos or ABs. Abs will be increased after prolonged exposure. MiRNAs are the main MV components which are altered robustly in each individual MV after hyperoxia. Multiple MV-miRNAs activate macrophages and subsequently exert pro-inflammatory effects, in a synergistic manner. 


\begin{tabular}{|l|l|l|}
\hline Name & primer sequence for $\mathbf{R T}\left(\mathbf{5}^{\prime}\right.$ to $\left.\mathbf{3}^{\prime}\right)$ & primer sequence for $\mathbf{q} \mathbf{P C R}\left(\mathbf{5}^{\prime}\right.$ to $\left.\mathbf{3}^{\prime}\right)$ \\
\hline Universal primer & & GGTGTCGTGGAGTCGGCAATTCAGTTGAG \\
\hline miR-221 & CTCAACTGGTGTCGTGGAGTCGGCAATTCAGTTGAGGAAACCCA & ACACTCCAGCTGGGAGCTACATTGTCTGCT \\
\hline miR-320a & CTCAACTGGTGTCGTGGAGTCGGCAATTCAGTTGAGTCGCCCTC & ACACTCCAGCTGGGAAAAGCTGGGTTGAG \\
\hline miR-342 & CTCAACTGGTGTCGTGGAGTCGGCAATTCAGTTGAGACGGGTGC & ACACTCCAGCTGGGTCTCACACAGAAATCG \\
\hline miR-92a & CTCAACTGGTGTCGTGGAGTCGGCAATTCAGTTGAGACAGGCCG & ACACTCCAGCTGGGTATTGCACTTGTCCC \\
\hline miR-422a & CTCAACTGGTGTCGTGGAGTCGGCAATTCAGTTGAGGCCTTCTG & ACACTCCAGCTGGGACTGGACTTAGGGTCA \\
\hline
\end{tabular}

Table 1. Sequences of primers used in qPCR.

\begin{abstract}
Materials and Methods
Materials. MiRNA-221 mimics (HMI0398), miRNA-320a mimics (HMI0470), miRNA-92a mimics (HMI0955), and miRNA-422a (HMI0562) were purchased from Sigma Aldrich (St. Louis, MO). Rabbit anti-phospho-p65 (Ser536) (3033), anti-p65 (8242) and anti-p50 (3035) were purchased from Cell Signaling (Danvers, MA). Human TNF-alpha DuoSet ELISA (DY210), Human IL-1 beta/IL-1F2 DuoSet ELISA (DY201), Human IL-10 DuoSet ELISA (DY217B), and Mouse TNF-alpha DuoSet ELISA (DY410) were purchased from $\mathrm{R} \& \mathrm{D}$ system (Minneapolis, MN).
\end{abstract}

Animals. Wild type C57BL/6 mice (male, 6 to 8 weeks of age) were obtained from Charles River Laboratories (Wilmington, MA, USA). All the protocols involving animals in this study were approved by the institutional animal care and use committee (IACUC) of Boston University. All experimental protocols and methods were approved by Boston University and were carried out in accordance with the approved guidelines.

Mouse primary cell isolation. Primary alveolar epithelial cells were isolated from mice as described previously $^{43}$. Briefly, mouse lung tissue was washed with sterile PBS, followed by infusion of $2 \mathrm{ml}$ dispase and $0.5 \mathrm{ml} 1 \%$ agarose. Lung tissue was then dissociated in DMEM with $25 \mathrm{mM}$ HEPES and $200 \mathrm{U} / \mathrm{ml}$ DNase. Isolated cells were sequentially passed through cell strainer (using $100 \mu \mathrm{m}, 40 \mu \mathrm{m}$ and $20 \mu \mathrm{m}$ cell strainer), followed by incubation on the plates pre-coated with CD 45 and CD16/32 antibodies for $2 \mathrm{~h}$. Suspended cells were further transferred to non-coated plates to get rid of fibroblast. After another $2 \mathrm{~h}$ incubation, suspended epithelial cells were culture in DMEM containing $10 \%$ FBS and subjected to further experiments.

Bone marrow-derived macrophages were isolated from mice as described previously ${ }^{44}$. Isolated bone marrow cells were cultured with $30 \%$ of L929-conditioned medium for 5 to 7 days to allow differentiation of the bone marrow monocyte/macrophage progenitors, and subjected to further experiments.

Cell culture. Human Beas2B lung epithelial cells and human THP1 monocytes were obtained from American Type Culture Collection (ATCC) and maintained in Dulbecco's modified Eagle's medium (DMEM), or RPMI1640 with $10 \%$ fetal bovine serum (FBS) and $1 \%$ penicillin/streptomycin. Cells were cultured at $37^{\circ} \mathrm{C}$ in a humidified atmosphere with $5 \% \mathrm{CO}_{2}$ and $95 \%$ air. For hyperoxia exposure, cells were exposed to hyperoxia (95\% oxygen with $5 \% \mathrm{CO}_{2}$ ) in modular exposure chambers, as previously described ${ }^{45}$.

Categorize the EVs. Three types of EVs were prepared by using sequential centrifugation protocols described previously with a minor modification ${ }^{23,46}$. Cultured cells were incubated with culture medium containing EV-depleted FBS for desired time points. Conditioned medium was collected and centrifuged at $300 \mathrm{~g}$ for $10 \mathrm{~min}$ to remove floating cells. The supernatant was further centrifuged at $2000 \mathrm{~g}$ for $20 \mathrm{~min}$ to pellet ABs. To isolate $\mathrm{MVs}$, the $\mathrm{AB}$-depleted supernatant was passed through a $0.8-\mu \mathrm{m}$-pore filter followed by centrifugation at $16,000 \mathrm{~g}$ for $40 \mathrm{~min}$. Finally, the resulting supernatant was passed through a $0.2-\mu \mathrm{m}$-pore filter and ultracentrifuged at 100,000 $\mathrm{g}$ for $1 \mathrm{~h}$ to pellet Exos. Each vesicles were further washed with cold PBS, then re-suspended with $\mathrm{PBS}$ and stored at $-80^{\circ} \mathrm{C}$. Protein concentration was measured with a Bradford assay.

RNA preparation, reverse transcription, and quantitative real-time PCR. Total RNAs were purified from isolated EVs using MiRNeasy Mini Kits (cat. no. 217004; Qiagen, Valencia, CA). Purified RNA amount was measured by NanoDrop Lite Spectrophotometer (Thermo Scientific). Reverse Transcription Kit (cat. no. 4374966, Thermo Fisher Scientific) was used to generate single stranded cDNA from the equal amount of purified RNAs. SYBR green-based real-time quantitative PCR (qPCR) technique was performed for detection of miRNAs as previously described ${ }^{47,48}$. The list of primers is shown in Table 1.

Electron microscopy. Purified EVs were fixed on the formvar-carbon coated mesh 400 grid according to the manufacturer's recommendation (101Bio, Palo Alto, CA). EV were visualized using transmission electron microscopy (Experimental Pathology Laboratory Core, Boston University School of Medicine).

Dynamic Light Scattering analysis. Size of EVs were analyzed using dynamic light scattering (DLS) instrument (Brookhaven 90plus Nano-particle Sizer, Biomedical Engineering core, Boston University). For calculating absolute EV number, standard calibration curve (particle number vs. count rate) was generated using DLS $\left(\mathrm{R}^{2}=0.9978\right)$. Count rate of each EV samples were measured by DLS, followed by calculation of absolute vesicle number. 
Quantitative analysis of MV contents. Total MV number, protein, and RNA amount (small and large RNAs) were obtained under RA and hyperoxia condition respectively. Next, we calculated the mean values of protein and RNA amount in single MV by dividing with MV counting number.

MiRNA microarrays. Three types of EVs were isolated from mouse BALF, followed by purification of total RNAs. The prepared RNA samples were analyzed with Multiplex miRNA Assay Immunology Panel (Firefly, ab204064, abcam). Microarray heatmap was generated using Firefly ${ }^{\circledR}$ Analysis Workbench software.

MiRNA transfection into MVs. Isolated MVs were incubated with Exo-Fect reagent [company?] and miRNA mimics $(10 \mathrm{pM})$ at $37^{\circ} \mathrm{C}$ for $10 \mathrm{~min}$ and placed on ice for $30 \mathrm{~min}$, followed by three times washing with PBS using 16,000 g-force centrifugation. Successful direct-transfection was confirmed using qPCR (not shown). The transfected MVs were directly used for further experiment.

Transwell migration assay. Macrophage migration analysis were performed using 6.5-mm-diameter polycarbonate 8-m microporous membranes (Costar, Cambridge, MA), as described previously ${ }^{49} .3 \times 10^{4}$ of the macrophages were placed on the inner chamber. EV-depleted $10 \%$ FBS was added in the outer well with miRNA-transfected MVs or miRNAs wrapped by lipofectamine 2000 (11668027, Thermo Fisher Scientific). After $16 \mathrm{~h}$ incubation, migrated cells were fixed with $4 \%$ formaldehyde and stained with hematoxylin. Migrated cells were counted using microscope.

ELISA assays. Macrophages were treated with miRNA-transfected MVs or miRNAs wrapped by lipofectamine 2000 for $16 \mathrm{~h}$, followed by collection of conditioned medium. TNF- $\alpha$, IL1 $\beta$, and IL10 cytokine levels were analyzed using DuoSet ${ }^{\circledR}$ ELISA Development Systems (R\&D system), according to the manufacturer's recommendation.

Gelatin Zymography. Prepared samples were loaded onto a SDS-PAGE containing $1 \mathrm{mg} / \mathrm{ml}$ gelatin and separated by electrophoresis under non-reducing condition. After washing with a $2.5 \%$ Triton X-100 for $1 \mathrm{~h}$, the gel was incubated in developing buffer containing $50 \mathrm{mM}$ Tris- $\mathrm{HCl}\left(\mathrm{pH} \mathrm{7.5)}\right.$ and $5 \mathrm{mM} \mathrm{CaCl}_{2}$ at $37^{\circ} \mathrm{C}$ for $16 \mathrm{~h}$. Gelatin lytic activity was analyzed by staining with Coomassie Brilliant Blue G-250.

Western Blot Analysis. Harvested cells were lysed with RIPA buffer containing 1\% triton X-100, protease inhibitor, and phosphatase inhibitor. Western blotting analysis was performed as described previously ${ }^{50}$.

Statistical analysis. All experiments were independently repeated 2-4 times. Represented data of the identical results were shown in the presented figures. The exact $n$ values of each experiments and statistical significances were shown in the corresponding figure legends. Statistical analysis was performed with unpaired two-tailed Student's T-test. Values of $\mathrm{p}<0.05$ were considered statistically significant $\left({ }^{\star} p<0.05,{ }^{* *} p<0.01\right)$.

\section{References}

1. Pitt, J. M., Kroemer, G. \& Zitvogel, L. Extracellular vesicles: masters of intercellular communication and potential clinical interventions. J Clin Invest 126, 1139-1143, doi: 10.1172/JCI87316 (2016).

2. Tkach, M. \& Thery, C. Communication by Extracellular Vesicles: Where We Are and Where We Need to Go. Cell 164, 1226-1232, doi: 10.1016/j.cell.2016.01.043 (2016).

3. Liu, Y. \& Cao, X. Organotropic metastasis: role of tumor exosomes. Cell Res 26, 149-150, doi: 10.1038/cr.2015.153 (2016).

4. Hoshino, A. et al. Tumour exosome integrins determine organotropic metastasis. Nature 527, 329-335, doi: 10.1038/nature15756 (2015).

5. Malda, J., Boere, J., van de Lest, C. H., van Weeren, P. R. \& Wauben, M. H. Extracellular vesicles - new tool for joint repair and regeneration. Nat Rev Rheumatol 12, 243-249, doi: 10.1038/nrrheum.2015.170 (2016).

6. Nawaz, M. et al. The emerging role of extracellular vesicles as biomarkers for urogenital cancers. Nat Rev Urol 11, 688-701, doi: 10.1038/nrurol.2014.301 (2014).

7. Martin, T. R. Cytokines and the acute respiratory distress syndrome (ARDS): a question of balance. Nat Med 3, 272-273 (1997).

8. Matthay, M. A., Ware, L. B. \& Zimmerman, G. A. The acute respiratory distress syndrome. J Clin Invest 122, 2731-2740, doi: 10.1172/JCI60331 (2012).

9. Whitsett, J. A. \& Alenghat, T. Respiratory epithelial cells orchestrate pulmonary innate immunity. Nat Immunol 16, 27-35, doi: $10.1038 /$ ni.3045 (2015)

10. Nold, M. F. et al. IL-37 is a fundamental inhibitor of innate immunity. Nat Immunol 11, 1014-1022, doi: 10.1038/ni.1944 (2010).

11. Lamm, W. J., Luchtel, D. \& Albert, R. K. Sites of leakage in three models of acute lung injury. J Appl Physiol (1985) 64, 1079-1083 (1988).

12. Li, X., Shu, R., Filippatos, G. \& Uhal, B. D. Apoptosis in lung injury and remodeling. J Appl Physiol (1985) 97, 1535-1542, doi: 10.1152/japplphysiol.00519.2004 (2004)

13. Jiang, D., Liang, J. \& Noble, P. W. Regulation of non-infectious lung injury, inflammation, and repair by the extracellular matrix glycosaminoglycan hyaluronan. Anat Rec (Hoboken) 293, 982-985, doi: 10.1002/ar.21102 (2010).

14. Moon, H. G. et al. Lung epithelial cell-derived extracellular vesicles activate macrophage-mediated inflammatory responses via ROCK1 pathway. Cell Death Dis 6, e2016, doi: 10.1038/cddis.2015.282 (2015).

15. Tao, F. \& Kobzik, L. Lung macrophage-epithelial cell interactions amplify particle-mediated cytokine release. Am J Respir Cell Mol Biol 26, 499-505, doi: 10.1165/ajrcmb.26.4.4749 (2002).

16. Matute-Bello, G., Frevert, C. W. \& Martin, T. R. Animal models of acute lung injury. Am J Physiol Lung Cell Mol Physiol 295, L379L399, doi: 10.1152/ajplung.00010.2008 (2008).

17. Divangahi, M., King, I. L. \& Pernet, E. Alveolar macrophages and type I IFN in airway homeostasis and immunity. Trends Immunol 36, 307-314, doi: 10.1016/j.it.2015.03.005 (2015).

18. Kopf, M., Schneider, C. \& Nobs, S. P. The development and function of lung-resident macrophages and dendritic cells. Nat Immunol 16, 36-44, doi: 10.1038/ni.3052 (2015).

19. Budnik, V., Ruiz-Canada, C. \& Wendler, F. Extracellular vesicles round off communication in the nervous system. Nat Rev Neurosci 17, 160-172, doi: 10.1038/nrn.2015.29 (2016). 
20. Thery, C., Ostrowski, M. \& Segura, E. Membrane vesicles as conveyors of immune responses. Nat Rev Immunol 9, 581-593, doi: $10.1038 /$ nri2567 (2009)

21. Lee, Y., El Andaloussi, S. \& Wood, M. J. Exosomes and microvesicles: extracellular vesicles for genetic information transfer and gene therapy. Hum Mol Genet 21, R125-R134, doi: 10.1093/hmg/dds317 (2012).

22. Xu, R., Greening, D. W., Zhu, H. J., Takahashi, N. \& Simpson, R. J. Extracellular vesicle isolation and characterization: toward clinical application. J Clin Invest 126, 1152-1162, doi: 10.1172/JCI81129 (2016).

23. Crescitelli, R. et al. Distinct RNA profiles in subpopulations of extracellular vesicles: apoptotic bodies, microvesicles and exosomes. J Extracell Vesicles 2, doi: 10.3402/jev.v2i 0.20677 (2013).

24. Aliotta, J. M. et al. Alteration of marrow cell gene expression, protein production, and engraftment into lung by lung-derived microvesicles: a novel mechanism for phenotype modulation. Stem Cells 25, 2245-2256, doi: 10.1634/stemcells.2007-0128 (2007).

25. Muralidharan-Chari, V. et al. ARF6-regulated shedding of tumor cell-derived plasma membrane microvesicles. Curr Biol 19, 1875-1885, doi: 10.1016/j.cub.2009.09.059 (2009).

26. Andreu, Z. \& Yanez-Mo, M. Tetraspanins in extracellular vesicle formation and function. Front Immunol 5, 442, doi: 10.3389/ fimmu.2014.00442 (2014).

27. Ratajczak, J. et al. Embryonic stem cell-derived microvesicles reprogram hematopoietic progenitors: evidence for horizontal transfer of mRNA and protein delivery. Leukemia 20, 847-856, doi: 10.1038/sj.leu.2404132 (2006).

28. Mittelbrunn, M. \& Sanchez-Madrid, F. Intercellular communication: diverse structures for exchange of genetic information. Nat Rev Mol Cell Biol 13, 328-335, doi: 10.1038/nrm3335 (2012).

29. Kogure, T., Yan, I. K., Lin, W. L. \& Patel, T. Extracellular Vesicle-Mediated Transfer of a Novel Long Noncoding RNA TUC339: A Mechanism of Intercellular Signaling in Human Hepatocellular Cancer. Genes Cancer 4, 261-272, doi: 10.1177/1947601913499020 (2013).

30. Ha, M. \& Kim, V. N. Regulation of microRNA biogenesis. Nat Rev Mol Cell Biol 15, 509-524, doi: 10.1038/nrm3838 (2014).

31. Zhang, L. et al. Microenvironment-induced PTEN loss by exosomal microRNA primes brain metastasis outgrowth. Nature 527, 100-104, doi: 10.1038/nature15376 (2015).

32. Tominaga, N. et al. Brain metastatic cancer cells release microRNA-181c-containing extracellular vesicles capable of destructing blood-brain barrier. Nat Commun 6, 6716, doi: 10.1038/ncomms7716 (2015).

33. Fong, M. Y. et al. Breast-cancer-secreted miR-122 reprograms glucose metabolism in premetastatic niche to promote metastasis. Nat Cell Biol 17, 183-194, doi: 10.1038/ncb3094 (2015).

34. Chevillet, J. R. et al. Quantitative and stoichiometric analysis of the microRNA content of exosomes. Proc Natl Acad Sci USA 111, 14888-14893, doi: 10.1073/pnas.1408301111 (2014).

35. Lo Cicero, A., Stahl, P. D. \& Raposo, G. Extracellular vesicles shuffling intercellular messages: for good or for bad. Curr Opin Cell Biol 35, 69-77, doi: 10.1016/j.ceb.2015.04.013 (2015).

36. Pospichalova, V. et al. Simplified protocol for flow cytometry analysis of fluorescently labeled exosomes and microvesicles using dedicated flow cytometer. J Extracell Vesicles 4, 25530, doi: 10.3402/jev.v4.25530 (2015).

37. Gong, Y., Hart, E., Shchurin, A. \& Hoover-Plow, J. Inflammatory macrophage migration requires MMP-9 activation by plasminogen in mice. J Clin Invest 118, 3012-3024, doi: 10.1172/JCI32750 (2008).

38. Wong, B. et al. Statins suppress THP-1 cell migration and secretion of matrix metalloproteinase 9 by inhibiting geranylgeranylation. J Leukoc Biol 69, 959-962 (2001).

39. Shubayev, V. I. et al. TNFalpha-induced MMP-9 promotes macrophage recruitment into injured peripheral nerve. Mol Cell Neurosci 31, 407-415, doi: 10.1016/j.mcn.2005.10.011 (2006).

40. Ji, H. et al. Deep sequencing of RNA from three different extracellular vesicle (EV) subtypes released from the human LIM1863 colon cancer cell line uncovers distinct miRNA-enrichment signatures. PLoS One 9, e110314, doi: 10.1371/journal.pone.0110314 (2014).

41. Gibbings, D. J., Ciaudo, C., Erhardt, M. \& Voinnet, O. Multivesicular bodies associate with components of miRNA effector complexes and modulate miRNA activity. Nat Cell Biol 11, 1143-1149, doi: 10.1038/ncb1929 (2009).

42. Choi, D. S., Kim, D. K., Kim, Y. K. \& Gho, Y. S. Proteomics of extracellular vesicles: Exosomes and ectosomes. Mass Spectrom Rev 34, 474-490, doi: 10.1002/mas.21420 (2015).

43. Liang, X. et al. p62 sequestosome 1/light chain $3 \mathrm{~b}$ complex confers cytoprotection on lung epithelial cells after hyperoxia. Am J Respir Cell Mol Biol 48, 489-496, doi: 10.1165/rcmb.2012-0017OC (2013).

44. Moon, H. G., Yang, J., Zheng, Y. \& Jin, Y. miR-15a/16 regulates macrophage phagocytosis after bacterial infection. J Immunol 193, 4558-4567, doi: 10.4049/jimmunol.1401372 (2014).

45. Jin, Y. et al. Caveolin-1 regulates the secretion and cytoprotection of Cyr61 in hyperoxic cell death. FASEB J 23, 341-350, doi: 10.1096/fj.08-108423 (2009).

46. Lazaro-Ibanez, E. et al. Different gDNA content in the subpopulations of prostate cancer extracellular vesicles: apoptotic bodies, microvesicles, and exosomes. Prostate 74, 1379-1390, doi: 10.1002/pros.22853 (2014).

47. Zhang, D. et al. Thyroid hormone regulates muscle fiber type conversion via miR-133a1. J Cell Biol 207, 753-766, doi: 10.1083/ jcb.201406068 (2014)

48. Chen, C. et al. Real-time quantification of microRNAs by stem-loop RT-PCR. Nucleic Acids Res 33, e179, doi: 10.1093/nar/gni178 (2005).

49. Lee, H. D., Kim, Y. H. \& Kim, D. S. Exosomes derived from human macrophages suppress endothelial cell migration by controlling integrin trafficking. Eur J Immunol 44, 1156-1169, doi: 10.1002/eji.201343660 (2014).

50. Lee, H. D., Koo, B. H., Kim, Y. H., Jeon, O. H. \& Kim, D. S. Exosome release of ADAM15 and the functional implications of human macrophage-derived ADAM15 exosomes. FASEB J 26, 3084-3095, doi: 10.1096/fj.11-201681 (2012).

\section{Acknowledgements}

This work is support by NIH R01HL102076 (Y.J.), NIH R21 AI121644 (Y.J.) and NIH R01 GM111313 (Y.J.). We thank Ms. Pawarissara Osathanugrah for her assistance on the sample preparation and cell culture.

\section{Author Contributions}

Y.J. conceived and supervised the project, Y.J. and C.S.D.C. wrote and revised the manuscript. H.L., D.Z. and Z.Z. designed the experiments, performed each individual assay, analyzed the data and wrote the manuscript. All authors reviewed the final manuscript.

\section{Additional Information}

Supplementary information accompanies this paper at http://www.nature.com/srep

Competing financial interests: The authors declare no competing financial interests. 
How to cite this article: Lee, H. et al. Epithelial cell-derived microvesicles activate macrophages and promote inflammation via microvesicle-containing microRNAs. Sci. Rep. 6, 35250; doi: 10.1038/srep35250 (2016).

(c) (i) This work is licensed under a Creative Commons Attribution 4.0 International License. The images

or other third party material in this article are included in the article's Creative Commons license, unless indicated otherwise in the credit line; if the material is not included under the Creative Commons license, users will need to obtain permission from the license holder to reproduce the material. To view a copy of this license, visit http://creativecommons.org/licenses/by/4.0/

(C) The Author(s) 2016 